\title{
TRANS-ABDOMINAL PREPERITONEAL AND TOTALLY EXTRAPERITONEAL LAPAROSCOPIC INGUINAL HERNIA REPAIR :A COMPARATIVE STUDY
}

\author{
Abd Al-Rahman Mohamed Hasanin Abd Al-Rahman Nawar (M.Sc), Tarek Ezat \\ Abd El-Latif (M.D), Wael El said Lotfy (M.D) \\ Department of general surgery, Faculty of medicine, Zagazig university, Egypt
}

\section{ABSTRACT}

Background: Laparoscopic repair of inguinal hernia repair has added to the ongoing debate over the "best groin hernia repair." There are two basic laparoscopic techniques, trans-abdominal pre-peritoneal (TAPP) and total extraperitoneal (TEP). Our study was carried out to compare these two methods of laparoscopic inguinal hernioplasty. Methods: This prospective randomized study was carried out in Surgery Department of Zagazig university hospitals between October 2013 and October 2015 and included 30 patients presenting with primary uncomplicated inguinal hernia. Patients were serially numbered and randomly arranged into two groups; group (A) as TAPP included 15 patients with odd number and group (B) as TEP included 15 patients with even number. All patients were admitted through the outpatient clinic and subjected to detailed history, clinical examination and laboratory work up. Results: Both groups were comparable regarding their demographic profile and hernia characteristics. The majority of hernias were indirect and left sided in both groups. The mean operative time was significantly longer in TAPP group compared to TEP group $(p=0.045)$. No major intraoperative complications were recorded apart from minor complications in the form of injury of the inferior epigastric artery $(p=0.67)$, pneumoscrotum $(p=0.62)$ and surgical emphysema $(p=0.67)$ with no significant difference between both groups. Postoperative pain scores recorded the day of surgery were significantly higher in TAPP group patients $(p=0.043)$ when compared to those in TEP group. They did not however differ significantly between the TEP and TAPP repairs in the 1 st postoperative day $(p=0.404)$. No persistent or serious postoperative complications were recorded apart from mild complications in the form of subcutaneous seroma in the early postoperative period $(p=0.794)$ with no significant difference between both groups. The mean postoperative hospital stay was significantly longer in TAPP group compared to TEP group $(p=0.033)$. The mean time interval before return to full activities was significantly longer in TAPP group compared to TEP group ( $p=0.038)$. Conclusion: Based on this study, laparoscopic TEP and TAPP repair of inguinal hernia is safe and efficacious. we can conclude that TEP was far better procedure compared to TAPP. According to mean operative time, post-operative pain, post-operative hospital stay and return to normal work, all were far better than TAPP.

Keywords: Laparoscopy, Total Extra-Peritoneal (TEP) repair, Trans Abdominal Pre Peritoneal (TAPP) repair, Inguinal hernia

Corresponding author: Abd Al-Rahman MH Nawar Received: November 2015

Mobile:+201006969780

E-mail address: abdelrahman.nawar@yahoo.com

Accepted: January 2016

\section{INTRODUCTION}

$\mathbf{P}$ robably no other disease entity could be treated with so many different procedures as groin hernia. This could be due to the fact that no single procedure is superior to the others. The development of technological and technical resources during the late 1980's allowed minimally invasive surgical interventions to be possible, including laparoscopic cholecystectomy, correction of gastroesophageal reflux disease, and groin hernia repair, among others ${ }^{[1]}$.
Over the last two centuries, a number of procedures had been developed for the repair of inguinal hernias, beginning with the Marcy repair and Bassini repair till the introduction of laparoscopic inguinal hernia repair adding to the ongoing debate over the "best groin hernia repair, ${ }^{[2]}$.

The advantages of laparoscopic inguinal hernia repair include less postoperative pain, short postoperative hospital stay, better 
cosmesis, early return to normal activity, and total absence of wound-related problems ${ }^{[1]}$.

There are two approaches of laparoscopic inguinal hernia repair, transabdominal preperitoneal repair (TAPP), described by Arregui and Doin in 1992, and total extraperitoneal repair (TEP), described by McKernan and Laws in $1993^{\text {[3] }}$

TAPP requires access to the abdominal cavity and the peritoneum over the posterior wall of the inguinal canal is incised with the placement of a large mesh in the preperitoneal space over all potential hernia sites. After fixation of the mesh, the peritoneum is carefully closed above the mesh ${ }^{[1]}$.

TEP is different in that the abdominal cavity is not entered without peritoneoscopy and a large mesh is placed over the myopectineal orifice This approach has gained ground in the past few years and is preferred over TAPP as it is less invasive and preserves the "peritoneal sanctity". However, TEP has a longer and steeper learning curve due to the "inside out anatomical view", to which the surgeon is not accustomed $^{[4]}$

Our study aimed to compare the results of both techniques of laparoscopic inguinal hernia repair; Total Extra- Peritoneal (TEP) and Trans Abdominal Pre-Peritoneal (TAPP) to determine if the relative advantages achieved could be put in practice in large scale and also identify criteria which may help stratify the patient to a particular type of repair to obtain encouraging results for that particular patient.

\section{PATIENTS AND METHODS}

This prospective randomized study was carried out in Surgery Department, Zagazig University Hospitals during the period from October 2013 through October 2015. The study included 30 patients presented with primary uncomplicated inguinal hernia for which they were operated upon by laparoscopic hernioplasty. The patients were serially numbered and randomly arranged into two groups; group (A) as TAPP included 15 patients with odd number and group (B) as TEP included 15 patients with even number.
All our patients were males, their age ranged between 18-55 years and diagnosed as having primary, uncomplicated, unilateral inguinal hernia. The following were excluded from our study :1.Patients with complicated inguinal hernia (irreducible, obstructed or strangulated). 2. Patients with huge or bilateral inguinal hernia. 3. Patients with recurrent inguinal hernia following laparoscopic repair. 4.Patients with relative or absolute contraindications for laparoscopy such as: marked obesity, coagulopathies, significant cardiopulmonary diseases or previous lower abdominal surgery.

All patients were admitted through the outpatient clinic and subjected to detailed history, clinical examination and laboratory work up

\section{Preoperative preparation}

1. A written valid consent was taken, 2.The side of hernia was marked. 3.NPO for at least 6 hours. 4.Shaving from xiphoid to groin and mid thigh. 5.The patient was asked to pass urine just before being shifted to the operating theatre.

\section{Operative procedure}

General endotracheal anesthesia is induced. The patient was placed supine in the Trendelenburg position with the arms tucked at the side. The surgeon stood on the opposite side of the hernia facing the pelvis while the assistant (camera operator) is positioned beside the surgeon at the shoulder of the patient. The video monitor is placed at the foot of the table (on the side of the hernia) (Fig. 1) . All patients had a single prophylactic dose of antibiotic at the induction of anaesthesia.

\section{Technique of TAPP repair}

Pneumoperitoneum up to $14 \mathrm{mmHg}$ was created with $\mathrm{CO} 2$ using a Veress needle at the umbilicus. A $10 \mathrm{~mm}$ trocar was placed through the supra-umbilical incision for introduction of the telescope. A 10-mm 30 degree telescope was used. Two operating 5-mm trocars were placed ; one below and lateral to the umbilicus in the midclavicular line on the epsilateral side of the hernia and the other $1 \mathrm{~cm}$ above the symphysis pubis in the midline (Fig.2). Exploration of the abdominal cavity was 
initially performed. Reduction of the inguinal hernia (if any) contents into the abdomen was done. The peritoneum was incised, at or about $2 \mathrm{~cm}$ above the deep ring, transversely from the medial umbilical ligament medially till the anterior superior iliac spine laterally to create an adequate peritoneal flap (Fig.3 $\boldsymbol{a} \& \boldsymbol{b})$. Dissection continued medially to the symphysis pubis to visualize the cave of Retzius identifying the shiny Cooper' $s$ ligament (Fig.4). The cord structures were identified after dissection of the peritoneal flap inferiorly. Adequate space was created for a $15 \times 15 \mathrm{~cm}$ polypropylene light mesh which was rolled and introduced via the $10 \mathrm{~mm}$ umbilical port into the space created. After unrolling the mesh and placing it over the entire myopectineal orifice on the affected side (covering the femoral, direct, and indirect sites of the hernia), it was fixed by tacks (The Tacker ${ }^{\mathrm{TM}} 5 \mathrm{~mm}$ Fixation Device). Fixation was started along the upper border of the mesh to the rectus muscle, at least $2 \mathrm{~cm}$ above the defect. The inferior edge was stapled to the symphysis pubis, Cooper's ligament medially and the iliopubic tract laterally (Fig.5).The peritoneal flap was closed using by using sutures (Vicryl2-0) to cover the mesh completely (Fig.6). It was helpful to decrease the intra-peritoneal pressure to less than $10 \mathrm{mmHg}$ for better approximation of the peritoneum. After completion of the surgery, desufflation of the abdominal cavity and scrotum if it was inflated, all instruments and sheathes were removed. No drains were left in any case. The skin cuts were closed subcutaneously using Vicryl 4-0 and steri-strips

\section{Technique of TEP repair}

A $2 \mathrm{~cm}$ subumbilical incision extending to the linea alba was made. The subcutaneous tissue was dissected carefully to expose the anterior rectus sheath (Fig.7). It was important to stay off the midline to avoid entering the peritoneal cavity. The anterior rectus sheath was incised transversely to expose the rectus muscle. The entire rectus muscle was retracted laterally to enter the space between the muscle and posterior rectus sheath. Using a finger and blunt dissection with $10 \mathrm{~mm}$ spoon instrument, a tunnel was made between the muscle and posterior rectus sheath for initial creation of the preperitoneal space (Fig.8). The balloon dissector device was prepared (A simple balloon tied securely with silk 0 around the tip of the guide rod while the other end connected to a pump) and tested before use (Fig.9). It was inserted beneath the rectus muscle toward the pubis and inflated to perform most of the preperitoneal dissection. Keeping the rectus muscle retracted laterally, the $10 \mathrm{~mm}$ sheath with its trocar were introduced obliquely into the space between muscle and the posterior rectus sheath for insertion of the telescope. A 10-mm 30 degree telescope was used. Two operating 5-mm trocars were placed; one introduced $4 \mathrm{~cm}$ below and lateral to the umbilicus on the epsilateral side of hernia and the other $1 \mathrm{crn}$ above symphysis pubis. The two operating ports were also placed in the midline in 3 cases : The first is placed approximately 1 $\mathrm{cm}$ above the symphysis pubis and the second halfway between the pubis and the periumbilical incision (Fig.10). The exposure of Cooper's ligament was begun with the dissection of the posterior aspect of the abdomina1 wall by gentle sweeping of the areolar tissue using alternate blunt and sharp dissection. The dissection proceeded laterally, identifying the inferior epigastric vessels, and further laterally up to the anterior superior iliac spine (Fig.11). The sac was completely reduced with gentle traction on the peritoneal attachments to the defect. In indirect inguinal hernia if the sac was large, it was circumferentially dissected, separated from the cord structures, severed and ligated proximally , leaving the distal sac in place. When the sac was completely dissected the pubic ramus and iliopubic tact were visualized on its entire extent. The femoral ring would be examined for possible femoral hernia. A light, $15 \times 15 \mathrm{~cm}$ sheet of polypropylene mesh was fashioned by cutting $5 \mathrm{~cm}$ slit vertically for the cord structures and introduced by rolling and stuffing it through the $10 \mathrm{~mm}$ trocar. The mesh was then unrolled to cover the entire myopectineal orifice on the affected side 
(covering the femoral, direct, and indirect sites of the hernia). The mesh was fixed by tacks (The Tacker ${ }^{\mathrm{TM}}$ 5mm Fixation Device) which were placed anterior to the iliopubic tract, cephalad and medial to the cord structures. The mesh was then stapled along Cooper's ligament and anterior abdominal wall (Fig.12). After inspecting all trocar sites and areas of dissection for homeostasis, desufflation of the extraperitoneal space was done. The skin cuts were closed subcutaneously using Vicryl 4-0 and steri- strips .

The following parameters were recorded and assessed 1. Intraoperative complications like vascular, nerve, visceral or vas injury. 2 . Operative time calculated in minutes from the time of incision till the time of wound closure.

\section{Post operative course}

The patient was observed in the recovery room for approximately 1 hour before transfer to the ward where further observation for 6-12 hours prior to discharge to home. Analgesics ( NSAIDs, I.M ) were given as needed, shifted to oral tablets ( Diclofenac potassium, $50 \mathrm{mg}$ ) twice daily. Patients encouraged to ambulate and start their activities of daily life as early as possible. Patients were observed and data recording for: 1. Post-operative complains such as subcutaneous emphysema, shoulder pain, scrotal swelling or testicular pain 2. Any complications such as seroma, hematoma or neuralgia.3. Postoperative pain using the Visual Analog Scale (VAS) which is a 10 points scale where 0 means no pain while 10 means the worst pain. Pain scores were recorded twice: D0 the day of surgery and D1 the 1st postoperative day. 4. Postoperative hospital stay in days. 5. The period, in days, required for the patient to resume his normal daily activities

Patients were discharged mostly within the next day or within 48 hours. At discharge they were advised to come after 7-8 days (1st follow up), then after 1week (2nd follow up), then after 1 month of surgery (3rd follow up). Later on after 3 months of surgery (4th follow up) and after 6 months of surgery (5th follow up)

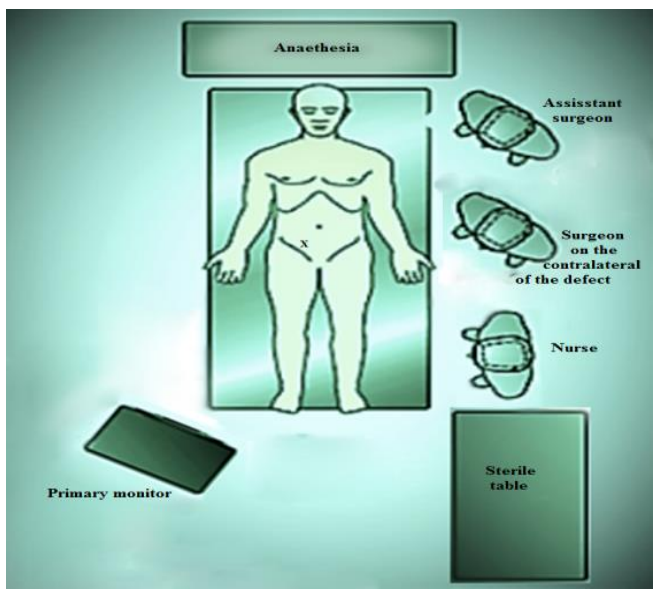

Fig.1 : Patient and team positioning in TAPP and TEP 

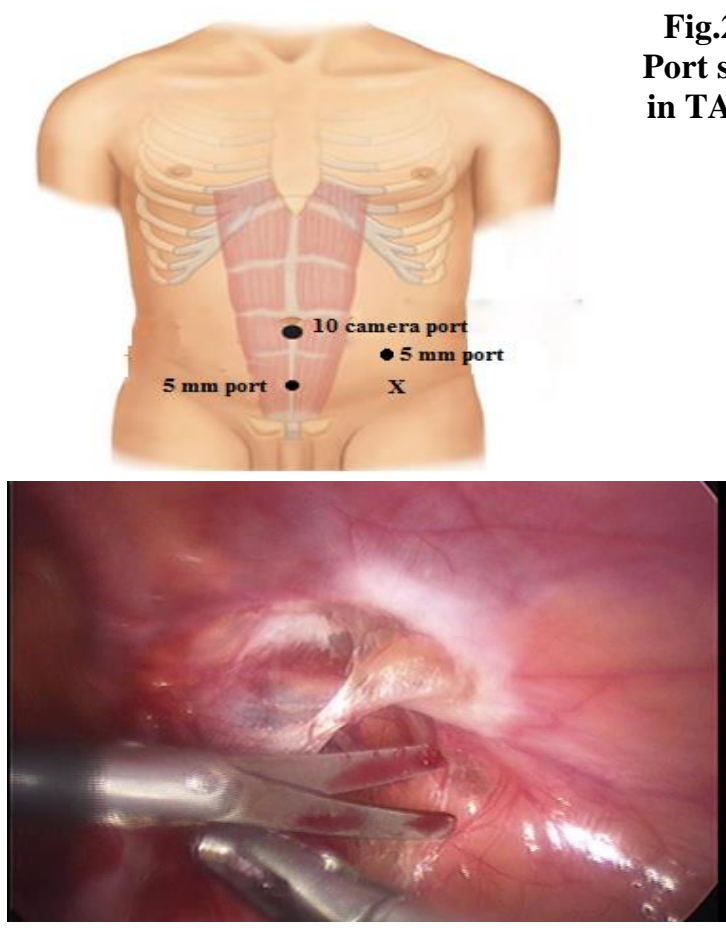

Fig. 3a : TAPP-1: Incision of the peritoneum Fig. 3b : TAPP-2: Extending the peritoneal incision

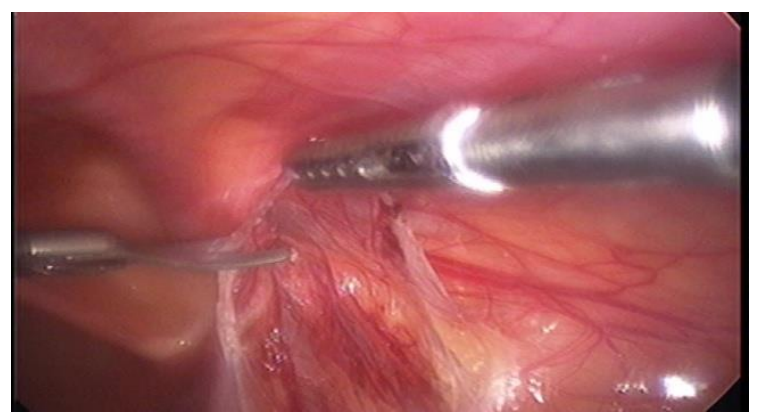

medially and laterally

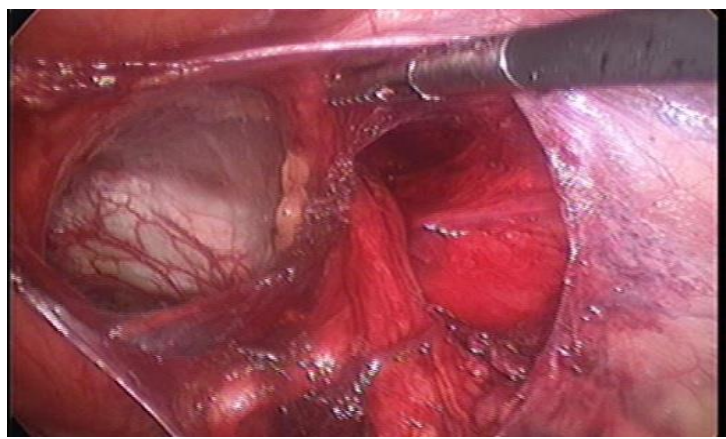

Fig. 4: TAPP-3: Identification of the epigastric vessels, internal inguinal ring, cooper's ligament, iliopubic tract, gonadal vessels and vas deferens

Fig.2 :

Port sites in TAPP

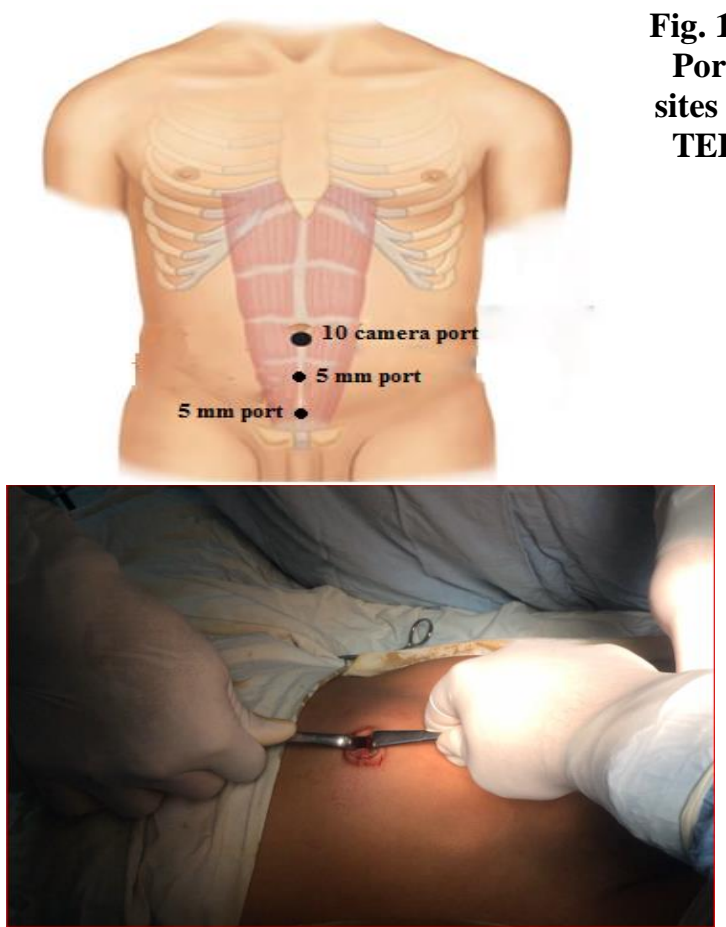

Fig. 7: TEP-1 Approaching the extraperitoneal space

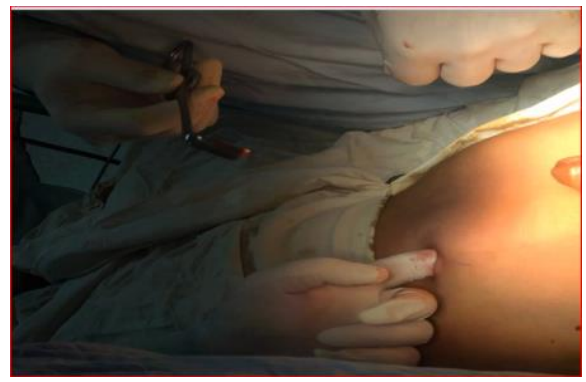

Fig. 8: TEP-2: Finger dissection of preperitoneal space

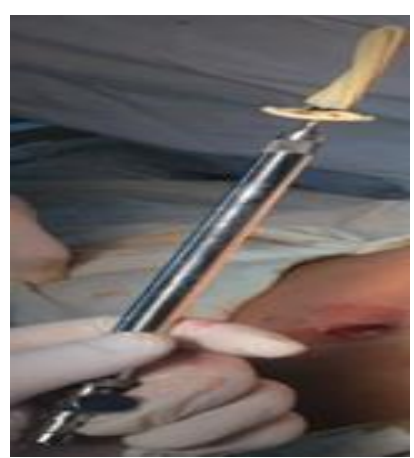

Fig. 9:TEP-3: Balloon dissector 


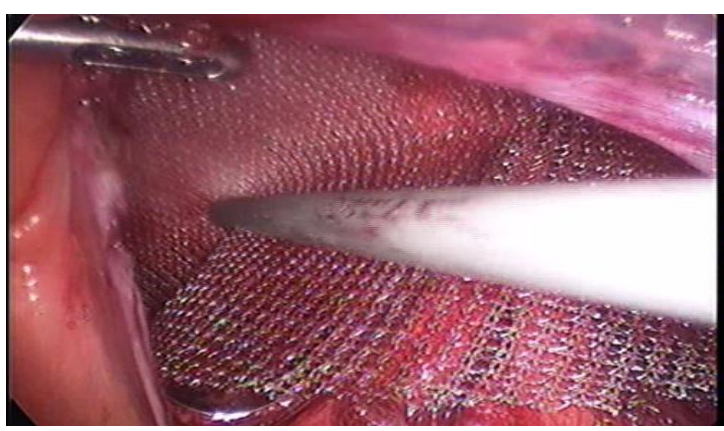

Fig. 5: TAPP-4: Fixation of mesh

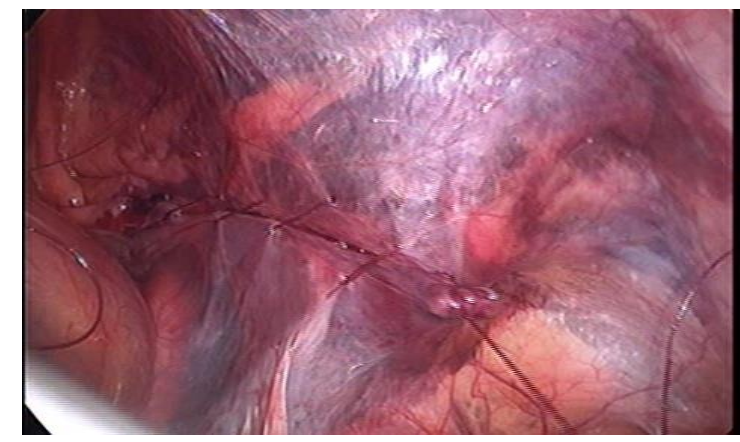

Fig. 6: TAPP-6: Closure of peritoneum over mesh

\section{STATISTICAL ANALYSIS}

All patients' data were collected, checked and analyzed by using (SPSS version 20). Data were expressed as mean \pm standard deviation (SD) and number with (\%) according to type of variable. Chi-square test $(\chi 2)$ or Fischer's exact $\mathrm{t}$ test or Mann-Whitney were used when appropriate. $\mathrm{P}$ value $<0.05$ was considered statistically significant.

\section{RESULTS}

This prospective randomized study was carried out in Surgery Department, Zagazig University Hospitals during the period from October 2013 through October 2015. The study included 30 patients presented with primary uncomplicated inguinal hernia for which they

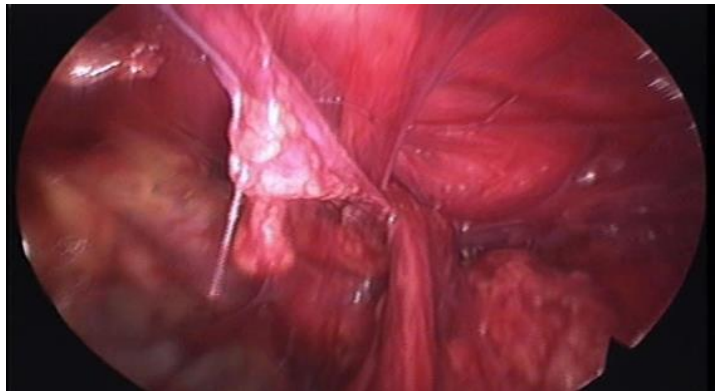

Fig. 11: TEP-4: Medial and lateral dissection of preperitoneal space

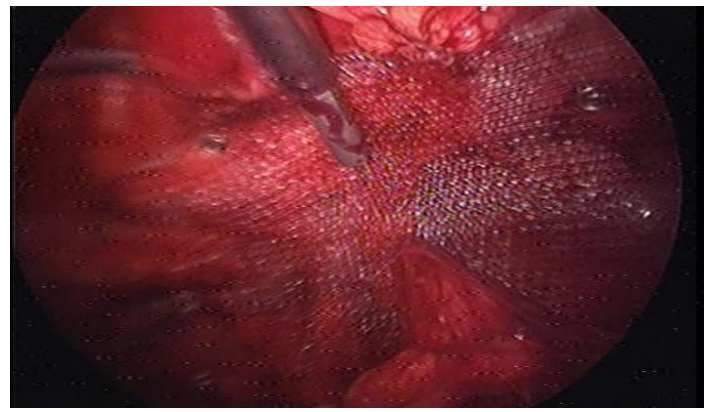

Fig. 12: TEP-5: Fixation of the mesh

were operated upon by laparoscopic hernioplasty. The patients were serially numbered and randomly arranged into two groups; group (A) as TAPP included 15 patients with odd number and group (B) as TEP included 15 patients with even number.

The groups were comparable, regarding their demographic profile (age, precipitating factors of hernia and co-morbidities) and hernia characteristics with no significant statistical difference

\section{Age distribution (Table 1):}

All the patients in our study were males. The mean age of patients in TAPP group was $30.2 \pm 9.872$ y $($ range $=18-55 \mathrm{y})$ while in TEP group was $30.13 \pm 9.657 \mathrm{y}($ range $=16-52 \mathrm{y})$. 
Table 1: Age distribution

\begin{tabular}{ccccccc}
\hline & & & & & \multicolumn{2}{c}{ Test of significance } \\
\cline { 2 - 5 } Age in years & Group $(A):$ TAPP & Group $(B):$ TEP & T test value & P value * \\
\cline { 2 - 5 } & No & $\%$ & No & $\%$ & & \\
\hline $20-29$ & 1 & 6.7 & 2 & 13.3 & \multirow{2}{*}{0.01} & \multirow{2}{*}{0.496} \\
\hline $30-39$ & 8 & 53.3 & 7 & 46.6 & \\
\hline $40-49$ & 3 & 20 & 4 & 26.7 & \\
\hline $50-59$ & 1 & 13.3 & 1 & 6.7 & \\
\hline Total & 15 & 6.7 & 1 & 6.7 & & \\
\hline
\end{tabular}

* As $P$ value is $>0.05$ so there is no statistical difference between the 2 groups regarding their age

\section{Precipitating factors of hernia (Table 2)}

Smoking was the commonest one; $6(40 \%)$ in TAPP group and 8(53.3\%) in TEP group while chronic cough ranked second to it ; $20 \%$ in TAPP group and $13.3 \%$ in TEP group. Three patients were suffering from chronic constipation; $2(13.3 \%)$ in TAPP group and $1(6.7 \%)$ in TEP group. Three patients gave history of lifting heavy objects ; 2 (13.3\%) in TAPP group and $1(6.7 \%)$ in TEP group.

Table 2: Precipitating factors of hernia

\begin{tabular}{|c|c|c|c|c|c|c|}
\hline \multirow{3}{*}{ Precipitating factors } & \multirow{2}{*}{\multicolumn{2}{|c|}{$\begin{array}{l}\underset{T A P P}{\operatorname{Group}}(A) \\
\operatorname{TAPP}\end{array}$}} & \multirow{2}{*}{\multicolumn{2}{|c|}{$\begin{array}{c}\operatorname{Group}(B): \\
\operatorname{TEP}\end{array}$}} & \multicolumn{2}{|c|}{ Test of significance } \\
\hline & & & & & \multirow{2}{*}{$X^{2}$} & \multirow{2}{*}{$P$ value ${ }^{*}$} \\
\hline & No & $\%$ & No & $\%$ & & \\
\hline Smoking & 6 & 40 & 8 & 53.3 & $\begin{array}{l}0.53 \\
5 \\
\end{array}$ & 0.745 \\
\hline Chronic constipation & 2 & 13.3 & 1 & 6.7 & 0.37 & 0.794 \\
\hline Chronic cough & 3 & 20 & 2 & 13.3 & 0.24 & 0.833 \\
\hline Lifting heavy objects & 2 & 13.3 & 1 & 6.7 & 0.37 & 0.794 \\
\hline No precipitating factors & 2 & 13.3 & 3 & 20 & 0.24 & 0.833 \\
\hline
\end{tabular}

*As $P$ value for all variables are $>0.05$ so there is no statistical difference between the two groups regarding the predisposing factors

\section{Co-morbidities (Table 3)}

Most patients; $13(86.6 \%)$ in TAPP group and $14(93.3 \%)$ in TEP group, had no co- morbidities while there were $2(13.4 \%)$ patients in TAPP group; 1 with hypertension and 1 with COPD, and $1(6.7 \%)$ patient in TEP group with COPD.) 
Table 3: Co-morbidities

\begin{tabular}{|c|c|c|c|c|c|c|c|}
\hline \multirow{2}{*}{\multicolumn{2}{|c|}{ Co-morbidities }} & \multicolumn{2}{|c|}{$\operatorname{Group}(A): T A P P$} & \multicolumn{2}{|c|}{ Group $(B): T E P$} & \multicolumn{2}{|c|}{ Test of significance } \\
\hline & & No & $\%$ & No & $\%$ & $X^{2}$ & $P$ value \\
\hline & Absent & 13 & 86.6 & 14 & 93.3 & \multirow{4}{*}{0.37} & \multirow{4}{*}{0.794} \\
\hline \multirow{3}{*}{ Present } & Hypertension & 1 & 6.7 & 0 & - & & \\
\hline & COPD & 1 & 6.7 & 1 & 6.7 & & \\
\hline & Total & 2 & 13.4 & 1 & 6.7 & & \\
\hline
\end{tabular}

*As P value is > 0.05 so there is no statistical difference between the two groups regarding the presence of associated disease

Characters of hernia (Table 4)

All our patients had primary unilateral hernias. The majority of hernias were left -sided [19 $(63.3 \%)]$; $10(66.7 \%)$ in TAPP group and 9 (60\%) in TEP group. Most hernias were indirect ;10 $(66.7 \%)$ in TAPP group and $9(60 \%)$ in TEP group and the other were direct ; $5(33.3 \%)$ in TAPP group and $6(40 \%)$ in TEP group.

Table 4: Characters of hernia

\begin{tabular}{|c|c|c|c|c|c|c|}
\hline \multirow{2}{*}{ Characters of hernia } & \multicolumn{2}{|c|}{$\operatorname{Group}(A): T A P P$} & \multicolumn{2}{|c|}{$\operatorname{Group}(B): T E P$} & \multicolumn{2}{|c|}{ Test of significance } \\
\hline & No & $\%$ & No & $\%$ & $X^{2}$ & Pvalue * \\
\hline Left & 10 & $66.7 \%$ & 9 & $60 \%$ & & \\
\hline Right & 5 & $33.3 \%$ & 6 & $40 \%$ & 0.143 & 0.862 \\
\hline Direct & 5 & $33.3 \%$ & 6 & $40 \%$ & & \\
\hline Indirect & 10 & $66.7 \%$ & 9 & $60 \%$ & & \\
\hline
\end{tabular}

* As $P$ value for all variables are > 0.05 so there is no statistical difference between the two groups regarding the Characters of hernia

Operative time in minutes (Fig. 1)

The mean operative time was ranged between $60-90$ with mean time of $74.2 \pm 8.612$ minutes for TAAP group and was ranged between $40-80$ with mean time of $57.53 \pm 9.403$ minutes for TEP group. So the mean operative time was significantly more in TAPP group when compared with the TEP group $(p=0.045)$. 
Fig. 1: Operative time in minutes

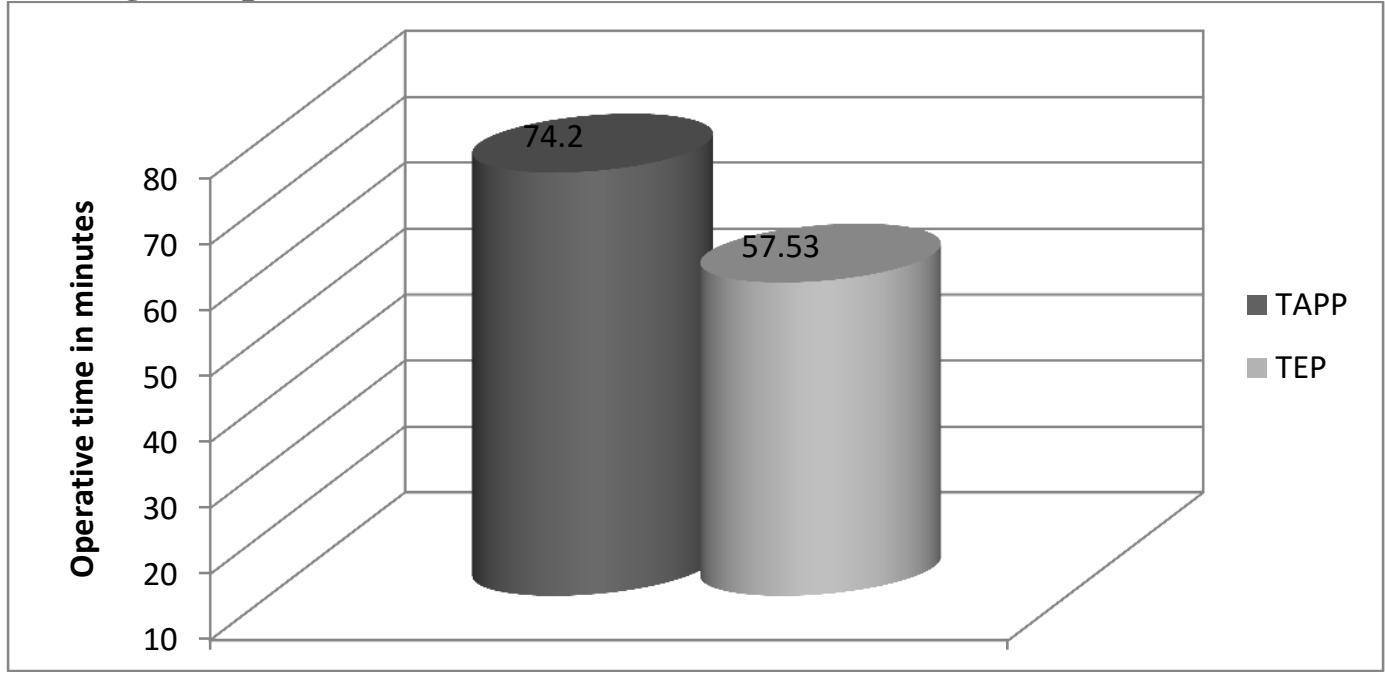

\section{Intra-operative complications (Table 5)}

In TAPP group there were 6 patients $(40 \%) ; 2$ patients with injury of inferior epigastric artery which were controlled with diathermy and clipping and 4 patients with pneumoscrotum which was managed by squeezing of the scrotum during desufflation. In TEP group, there were 3 patients $(20 \%)$; one patient developed pneumoscrotum which was managed by the same way and 2 cases with surgical emphysema which was self-limited and resolved by 2 nd postoperative day. This difference was not statistically significant $(p=0.48)$ between the two groups.

Table 5: Intra-operative complications

\begin{tabular}{ccccccc}
\hline & & & & & \multicolumn{2}{c}{ Test of significance } \\
\cline { 3 - 6 } Intra-operative complications & Group $(A):$ TAPP & Group $(B):$ TEP & & $X^{2}$ & P value * \\
\cline { 2 - 6 } & No & $\%$ & No & $\%$ & & 0.67 \\
\hline $\begin{array}{c}\text { Injury of inferior epigastric } \\
\text { artery }\end{array}$ & 2 & 13.3 & 0 & - & 2.14 & 0.62 \\
\hline $\begin{array}{c}\text { Pneumoscrotum } \\
\text { Surgical emphysema }\end{array}$ & 4 & 26.7 & 1 & 6.7 & 2.16 & 0.67 \\
\hline $\begin{array}{c}\text { Total number for } \\
\text { complications }\end{array}$ & 6 & - & 2 & 13.3 & 2.14 & 0.48 \\
\hline
\end{tabular}

* As $P$ value for all variables are $>0.05$ so there is no statistical difference between the two groups regarding the intra-operative complications

\section{Postoperative pain (Fig. 2)}

Pain score in TAPP group in the day of surgery (day 0) was 1-2 (mild) in 12 patient (80\%) and 3-6 (moderate) in 3 patients (20\%) [2.6 \pm 1.298$]$ while in TEP group it was $1-2$ in 14 patient $(93.3 \%)$ and $3-6$ in 1 patient $(6.7 \%)$ [1.467 \pm 0.64$]$. So there was a significant difference $(p=0.043)$ between the two groups regarding the postoperative pain in the same day of surgery. In the 1st postoperative day, pain 
score in TAPP group was 1-2 (mild) in 14 patient $(93.3 \%$ ) and 3-6 (moderate) in one patient $(6.7 \%)$

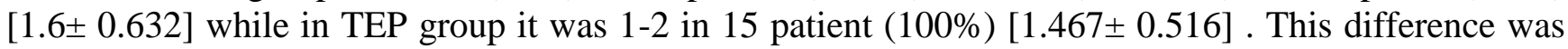
of no statistical significance $(\mathrm{p}=0.404)$

Fig. 2: Postoperative pain

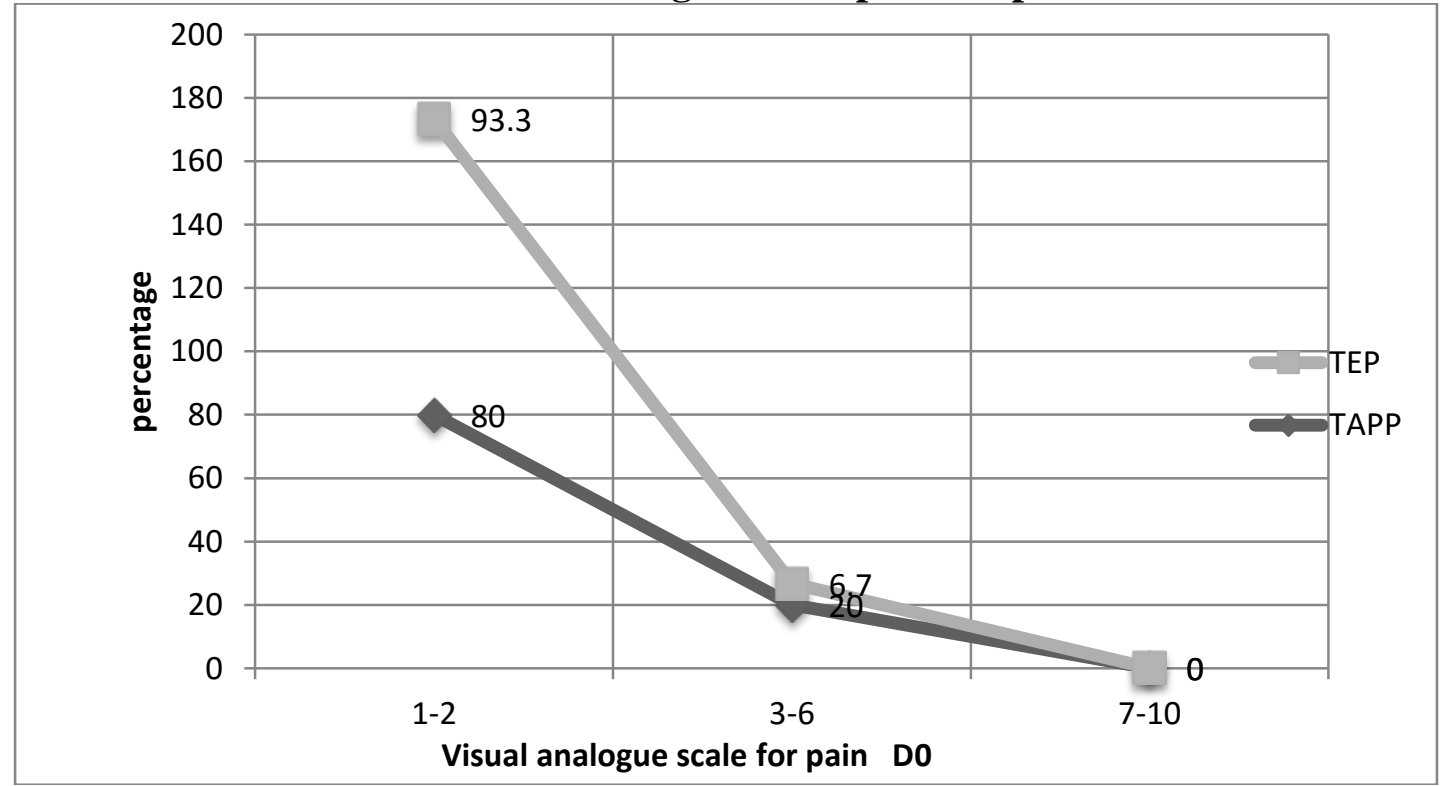

\section{Postoperative complications}

No persistent or serious complications were recorded in our study apart from mild complications. Two patients developed subcutaneous seroma in TAPP group (13.3\%) which were self limited and treated conservatively while in TEP group, there was one patient who developed similar seroma (6.7\%) and treated also conservatively. This difference was not statistically significant $(\mathrm{p}=0.794)$ between the two groups.

Postoperative hospital stay in hours (Fig. 3)

All cases of both groups were discharged in less than 2 days postoperatively. However in TAPP group, the mean postoperative hospital stay was $24.53 \pm 4.642 \mathrm{~h}$ which was significantly longer that of TEP group, $13.67 \pm 5.486 \mathrm{~h}(p=0.033)$

Fig. 3: Postoperative hospital stay

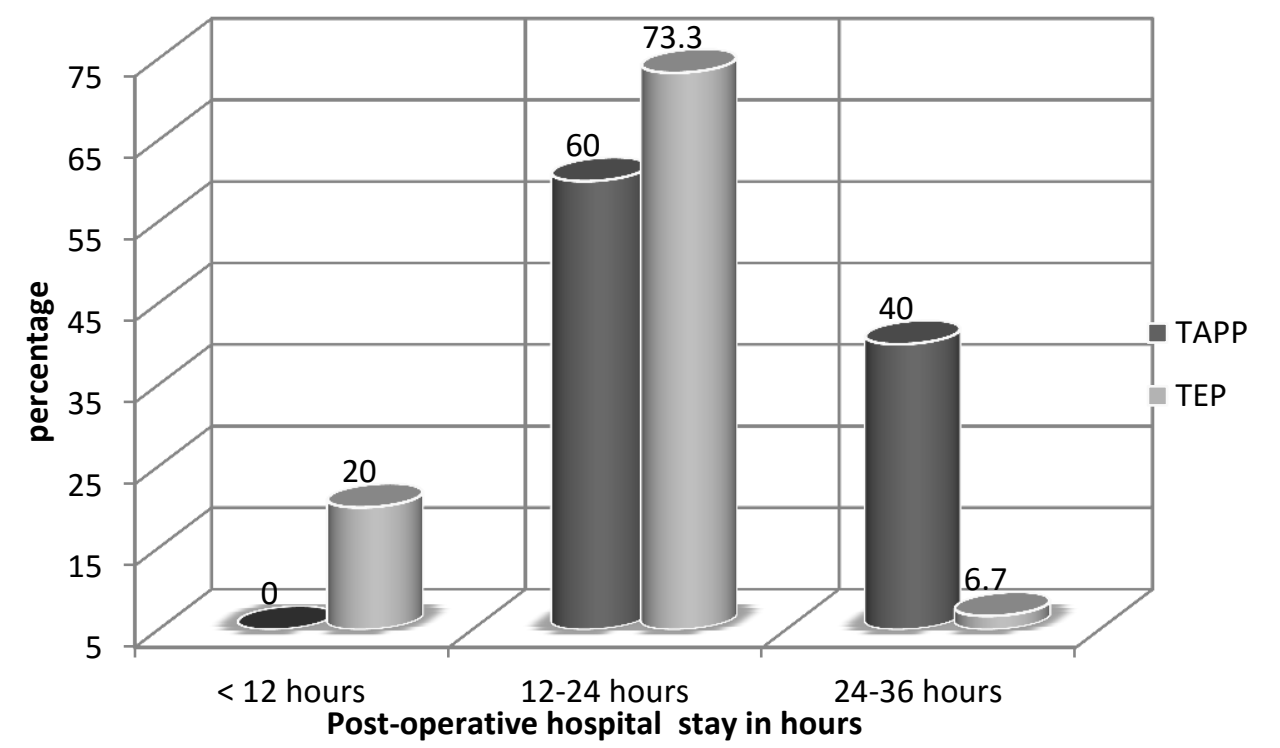


Time interval before return to full activities in days (Table 6)

The mean time interval before return to full activities in TAPP group was $12 \pm 1.464$ days while in TEP group was $9.067 \pm 1.58$ days $(p=0.038)$. So there is statistically significant difference between the two groups in the favour of TEP procedure with early return to work. This may be due to lower pain scores recorded in TEP group.

Table 6: Time interval before return to full activities in days

Test of significance

Time interval (days)

$\operatorname{Group}(A): T A P P \quad G r o u p(B): T E P$

\begin{tabular}{|c|c|c|c|c|c|c|}
\hline & & & & & \multirow{2}{*}{$T$ test } & \multirow{2}{*}{ Pvalue ** } \\
\hline & No & $\%$ & No & $\%$ & & \\
\hline $7-13$ & 3 & 20 & 14 & 93.3 & \multirow{3}{*}{-1.86} & \multirow{3}{*}{0.038} \\
\hline $14-21$ & 12 & 80 & 1 & 6.7 & & \\
\hline Mean $\pm S D$ & \multicolumn{2}{|c|}{$12 \pm 1.464$} & \multicolumn{2}{|c|}{$9.067 \pm 1.58$} & & \\
\hline
\end{tabular}

\section{DISCUSSION}

Inguinal hernia repair is one of the most common elective general surgical operations. However there has been no universal agreement on the ideal repair, which would avoid the potential problems of wound infection, recurrence and chronic groin pain

There are two types of laparoscopic repair of inguinal hernia namely laparoscopic TEP repair and laparoscopic TAPP repair, both of which have evolved over a period of just two decades with a distinct conflict regarding the superiority of one over the other.

The present study aimed to compare TAPP and TEP and evaluate each technique for proper selection of each in laparoscopic treatment of inguinal hernia.

Regarding the operative time, our mean time of operation in TAPP group was $74.2 \pm$ $8.612 \mathrm{~min}$ (range $=60-90 \mathrm{~min}$ ) while in TEP group was $57.53 \pm 9.403 \mathrm{~min}$ (range $=40-80$ min). Hence the overall mean operative time was significantly less in laparoscopic TEP repair than in laparoscopic TAPP repair. This longer operative time for TAPP in our study due to the time taken for closing the peritoneum to cover the mesh. On average, it took about 8 9 min

These data were comparable to those found in the study performed by Krishna and his colleagues (2012) ${ }^{[5]}$ as the mean operative time in TAPP group is $72.3 \pm 25.9 \mathrm{~min}$ (range $=30$ $130 \mathrm{~min}$ ) while in TEP group is $62.1 \pm 20.6 \mathrm{~min}$ (range $=35-120 \mathrm{~min}$ ).

However, our data are in contrary to those found in the study of Ashraf Zeineldin (2008) [6] in which the mean operative time in TAPP group was $43 \pm 11.3$ min while in TEP group was $57.3 \pm 15.7 \mathrm{~min}$. The author stated that TAPP gives a good view and surgeons get oriented with the anatomical structures quickly. It also takes shorter time to perform specially in the hands of surgeons who are not very experienced and new learners.

In the study performed by Hamza and his colleagues (2009) ${ }^{[7]}$, the mean operative time in TAPP group was $96.12 \pm 22.5 \mathrm{~min}$ while in TEP group was $77.4 \pm 43.21$ min which were longer than our results. As the setup time was 
included, the operating time became further elongated.

Regarding the intra-operative complications, in TAPP group there were 6 patients (40\%) ;2 patients with injury of inferior epigastric artery which were controlled with diathermy and clipping and 4 patients with pneumoscrotum which was managed by squeezing of the scrotum during desufflation. In TEP group, there were 3 patients $(20 \%)$; one patient developed pneumoscrotum which was managed by the same way and 2 cases with surgical emphysema which was self-limited and resolved by 2 nd postoperative day. This difference was not statistically significant $(\mathrm{p}=0.48)$ between the two groups.

Our results were nearly similar to those of the study performed by Choksi and his colleagues (2014) ${ }^{[8]}$ on comparing the TAPP and TEP, in which there were 6 patients $(26.66 \%)$ with intraoperative complications in TAPP repair ; 2 patients with injury of inferior epigastric artery, one patient with injury of the vas deferens and 3 patients with pneumoscrotum while in TEP repair, they recorded intraoperative complications in 8 patients (20\%); 2 patients with injury of inferior epigastric artery, 2 patients were converted to TAPP, one patient was converted to open repair and 3 patients with pneumoscrotum.

Also our results were nearly similar to those of the study of Ashraf Zeineldin (2008) ${ }^{[6]}$, in which there were two injuries of the inferior epigastric artery in the TAPP group (2.9\%). The vessel was clipped laparoscopically. Two TEP procedures were converted to TAPP approach due to anatomical difficulties (3.3\%).

Postoperative pain was assessed using visual analogue scale (Score 1 to 10 ). In our study, pain score in TAPP group in the day of surgery (day 0) was 1-2 (mild) in 12 patient $(80 \%)$ and 3-6 (moderate) in 3 patients $(20 \%)$ [2.6 \pm 1.298$]$ while in TEP group it was $1-2$ in 14 patient $(93.3 \%)$ and $3-6$ in 1 patient $(6.7 \%)$ $[1.467 \pm 0.64]$. So there was a significant difference $(\mathrm{p}=0.043)$ between the two groups regarding the postoperative pain in the same day of surgery. In the 1st postoperative day , pain score in TAPP group was 1-2 (mild) in 14 patient $(93.3 \%)$ and 3-6 (moderate) in one patient $(6.7 \%)$ [1.6 \pm 0.632$]$ while in TEP group it was $1-2$ in 15 patient $(100 \%)$ [1.467 \pm 0.516$]$. This difference was of no statistical significance $(\mathrm{p}=0.404)$.

These data were comparable to those found in the study of Krishna and his colleagues (2012) ${ }^{[5]}$ as there were higher pain scores at 12 $h$ in TAPP group compared to TEP group. Pain score at $12 \mathrm{~h}$ in TAPP group was $2.79 \pm 0.55$ while in TEP group was $1.98 \pm 0.24$. The difference between the two groups was statistically highly significant $(\mathrm{p}=0.0001)$. After $24 \mathrm{~h}$ patients in TAPP group had higher pain scores $(2.21 \pm 0.549)$ than those in TEP group $(1.47 \pm 0.541)$. However, the difference between the two groups was not statistically significant $(\mathrm{p}=0.108)$.

In the study performed by Hamza and his colleagues (2009) ${ }^{[7]}$, statistical significance was found at $12 \mathrm{~h}$ and after $24 \mathrm{~h}$ regarding pain scores between TAPP and TEP groups .In TAPP group pain score at $12 \mathrm{~h} 5.8 \pm 1.568$ while in TEP group it was $4.8 \pm 2.33(\mathrm{p}=0.002)$. After 24, pain score was $4.133 \pm 1.125$ in TAPP group while in TEP group it was $3.98 \pm 4.35$ ( $\mathrm{p}=$ 0.020 ) and these results were nearly similar to ours.

Regarding the postoperative complications, no persistent or serious complications were recorded in our study apart from mild complications. Two patients developed subcutaneous seroma in TAPP group (13.3\%) which were self limited and treated conservatively while in TEP group, there was one patient who developed similar seroma $(6.7 \%)$ and treated also conservatively. This difference was not statistically significant $(p=0.794)$ between the two groups.

The postoperative complication results were comparable to those found in the study of Verma and his colleagues (2015) ${ }^{\text {[9] }}$ where no statistical significant difference was found regarding his postoperative complications. In TAPP group, there were 5 patients developed mild complications $(16.7 \%)$ [ two patients with 
port site infection, one patient with right shoulder pain and 2 patients had early transient groin pain] while in TEP group of his study, there were 6 patients suffered also mild complications $(20 \%)$ [ one patient had port site infection, another patient developed early transient groin pain, 2 patients had subcutaneous seroma and 2 others developed hematoma at the port site].

According to results obtained by Choksi and his colleagues (2014) ${ }^{[8]}$, there were 8 patients with minor complications among the TEP group (26.66\%) compared to 6 patients among the TAPP group $(20 \%)(\mathrm{p}=0.54155)$. Also in Hamza's study (2009) ${ }^{[7]}$, there were 4 patients $(16 \%)$ in TAPP group with minor complications [ one patient had scrotal hematoma, another patient had wound infection, one developed groin pain while the last one had recurrence of his hernia] while in TEP group , there was only one patient with recurrence $(p=0.425)$. These data were comparable to those found in our study with no statistical significant difference regarding the postoperative complications between the two groups, although we didn't have recurrence of hernia among our patients, but still the incidence of this complication is low.

However our results were different from those reported in the study performed by Krishna and his colleagues (2012) ${ }^{[5]}$ where the scrotal edema incidence was significantly higher in group TAPP $(\mathrm{p}=0.009)$. Five patients (9.4\%) among the TEP group and 16 patients (34\%) among the TAPP group developed scrotal edema in the immediate postoperative period. This higher incidence of scrotal edema could be related to the higher rate of patients with indirect hernias in the TAPP group $(70.2 \%$ ) and also most of the patients who developed scrotal edema had a large hernial sac.

In the same study, The seroma formation rate was significantly higher in the TEP group ( $\mathrm{p}=0.021)$. At 7-day follow-up, 20 patients $(37.9 \%)$ in TEP group and 8 patients $(17 \%)$ in TAPP group developed seroma. Seroma resolved in 23 patients by the end of first month on expectant management. Five patients (3 in
TEP group and 2 in TAPP group) had persistent seroma at one-month follow-up requiring aspiration. This higher incidence of seroma was among old age patients, those with large hernial defect or extension of the hernia into the scrotum and keeping the distal part of indirect hernia sac in-situ.

Regarding the postoperative hospital stay, all cases of both groups were discharged in less than 2 days postoperatively. In TAPP group, the mean postoperative hospital stay was $24.53 \pm 4.642 \mathrm{~h}$ while in TEP group, it was $13.67 \pm 5.486 \mathrm{~h}$. As observed from these data, $\mathrm{p}$ value was $<0.05$ so the difference was statistically significant between the two groups in the favour of TEP procedure.

Our results were nearly similar to those in the study of Krishna and his colleagues (2012) [5] although there was no statistical significance $(\mathrm{p}=0.056)$. regarding postoperative hospital stay between the two groups. However, in TEP group the mean postoperative stay was $24.4 \pm$ $3.2 \mathrm{~h}$ which was shorter than that in TAPP group, $25.2 \pm 5.1 \mathrm{~h}$.

However our results were different from those reported in the study performed by Choksi and his colleagues (2014) ${ }^{[8]}$ in which the mean duration of post-operative hospital stay was $2.8 \pm 1.3$ days in TEP group while it was $2.76 \pm 1.0$ days in TAPP group which was not statistically significant between two groups $(\mathrm{p}=0.70595)$.

Concerning the time interval before return to full activities of daily life, in our study the mean time interval in TAPP group was $12 \pm$ 1.464 days while in TEP group was $9.067 \pm$ 1.58 days $(\mathrm{p}=0.038)$. So there is statistically significant difference between the two groups in the favour of TEP procedure with early return to work. This may be due to lower pain scores recorded in TEP group.

Similarly statistical significance $(p=0.001)$ was found on looking to the results of Hamza and his colleagues (2009) ${ }^{[7]}$, in their comparative study between the TAPP and TEP, where the mean time interval before return to activity in TAPP group was 9.8 5.979 days while in TEP group it was $7.53 \pm 3.65$ days. 
However, there was no statistical significance $(\mathrm{p}=>0.05)$ in the study performed by Verma and his colleagues (2015) ${ }^{\text {9] }}$ in which the mean time interval before return to activity in TAPP group was 7.2 \pm 2.31 while in TEP group it was $6.43 \pm 2.76$. The results of both studies of Hamza and Verma were comparable to our results.

\section{CONCLUSION}

Based on this study, laparoscopic TEP and TAPP repair of inguinal hernia is safe and efficacious. we can conclude that TEP was far better procedure compared to TAPP. According to mean operative time, post-operative pain, post-operative hospital stay and return to normal work, all were far better than TAPP.

\section{REFERENCES}

1. Carter J and Duh QY : Laparoscopic Repair of Inguinal Hernias. World J Surg, 2011; 35:1519-1525

2. Kulacoglu $\mathbf{H}$ : Current options in inguinal hernia repair in adult patients. HIPPOKRATIA, 2011; 15(3) : 223-231

3. Davis CJ and Arregui ME : Laparoscopic repair for groin hernias. Surg. Clin. North. Am, 2003; 83: 1141-1161.

4. McCormack B L, Wake $C$ and Fraser L : Transabdominal pre-peritoneal (TAPP) versus totally extraperitoneal (TEP) laparoscopic techniques for inguinal hernia repair: a systematic review. Hernia, 2005; 9: 109-114.

5. Krishna A , Misra $M$ and Bansal VK : Laparoscopic inguinal hernia repair: transabdominalpreperitoneal (TAPP) versus totally extraperitoneal (TEP) approach: a prospective randomized controlled trial. Surg Endosc, 2012 ; 26:639-649

6. Zeineldin A : Transabdominal preperitoneal (TAPP) versus totally extraperitoneal (TEP) laparoscopic inguinal hernia repair: A prospective comparative study, 2008; Vol. 21 (2): 147-154

7. HamzaY, Gabr E, Hammadi $H$ and Khalil R : Four arm randomized trial comparing laparoscopic and open hernia repairs. Egyptian Journal of Surgery, 2009; Vol 28( 3): 110-117.

8. Choksi D, Parmar A and Raiyani G: Comparative prospective study of laparoscopic TEP repair versus laparoscopic TAPP repair for inguinal hernioplasty conducted at tertiary level hospital, Vadodara, Int J Res Med , 2014; 3(1): 17-19

9. Verma N, Prajapati $K$ and Mistry $S$ : A comparative study between total extraperitoneal (TEP) repair and Transabdominal pre-peritoneal (TAPP) repair in management of inguinal hernia . National Journal of Medical Research, 2015; Vol 5 :64-66 